\title{
Revisión del estado taxonómico de Nyctelia multicristata Blanchard, 1846 y descripción de Nyctelia confusa Zuñiga-Reinoso n. sp. (Coleoptera: Tenebrionidae)
}

\section{Review of the Taxonomic status of Nyctelia multicristata Blanchard, 1846 and description of Nyctelia confusa Zuñiga-Reinoso sp. n. (Coleoptera: Tenebrionidae)}

\author{
Álvaro Zúñiga-Reinoso ${ }^{1 *} \&$ Viviane Jerez ${ }^{1}$ \\ 1'Departamento de Zoología, Facultad de Ciencias Naturales y Oceanográficas. Universidad de Concepción. \\ *Email: alzure@gmail.com
}

\begin{abstract}
RESUMEN
Existe una confusión con la validez taxonómica de Nyctelia multicristata Blanchard en Hombron \& Jaquinot (1846). Esto se debe a que Kulzer (1963) en la revisión del género Nyctelia, redescribe a la especie N. multicristata y describe una nueva especie, $N$. fallax, en base a caracteres morfológicos poco claros y la mayoría compartidos con $N$. multicristata. El análisis morfológico, reveló que los especimenes adscritos a $N$. fallax se debe sinonimizar con $N$. multicristata. Tanto la morfología externa y la genitalia revelan que el morfotipo N. multicristata redescrito por Kulzer, corresponde a una nueva especie.
\end{abstract}

Palabras claves: Nycteliini, Nyctelia fallax, sinonimia, Nyctelia confusa, taxonomía.

\begin{abstract}
There is confusion with the taxonomic validity of Nyctelia multicristata Blanchard in Hombron \& Jaquinot (1846). This is because Kulzer (1963) in the review of the genus Nyctelia, redescribed the species N. multicristata and described a new species, $N$. fallax, based on unclear morphological traits which most are shared with $N$. multicristata. Morphological analysis revealed that specimens assigned to $N$. fallax must be synonymized with $N$. multicristata. Both the external morphology and genitalia reveals that the morphotype $N$. multicristata described by Kulzer corresponds to a new species.
\end{abstract}

KeYwords: Nyctelini, Nyctelia fallax, synonymy, Nyctelia confusa, taxonomy

\section{INTRODUCCIÓN}

El género neotropical Nyctelia Latreille, 1825 (Tenebrionidae: Pimeliinae: Nyctelini), comprende 64 especies descritas, distribuidas desde el noroeste de Argentina y Chile central hasta el extremo sur de Patagonia (Flores 1997, Flores \& Carrara 2006, Flores 2007, Flores 2009). Este género es endémico de biomas áridos y semiáridos del Sur de Sudamérica, tanto de desiertos como de altamontaña (Flores 1997, Roig-Juñent \& Flores 2001, Flores \& Carrara 2006, Vidal \& Guerrero 2007), son especialmente abundantes en la estepa Patagónica, pudiendo encontrarse varias especies en simpatria (Peña 1963, Flores \& Carrara 2006). Sin embargo y a pesar de su abundancia, no existen estudios sobre su biología (i.e etología, ecología y desarrollo) y aún persisten problemas taxonómicos con algunos grupos de especies, particularmente aquellas distribuidas en el extremo sur de Sudamérica.
Un ejemplo de ello, es el caso del estado taxonómico de Nyctelia multicristata Blanchard en Hombron \& Jaquinot 1846, dado que desde su descripción esta especie se ha transformado en un caso conflictivo desde el punto de vista taxonómico. Kulzer (1963) en su revisión de las Nyctelia, estableció el status taxonómico de cada especie y las diferencias interespecíficas, en basea caracteres morfológicos externos. En esta revisión, Kulzer (1963) describió Nyctelia fallax, especie muy parecida morfológicamente a $N$. multicristata, pero los caracteres morfológicos que diferencian ambas especies son poco claros, ya que los caracteres diagnósticos entregados por Kulzer (1963) para N. fallax, son similares a los descritos por Solier (1851: 141) y Blanchard (1853: 143) para $N$. multicristata. Es probable que por este motivo, en publicaciones posteriores $\mathrm{y}$ en colecciones entomológicas, se puede comprobar que existen errores de identificación entre ambas especies (e.g Arias 2000, Vidal \& Guerrero 2007). Sin embargo, también 
existe una confusión con el año y el nombre del autor de la especie $N$. multicristata, el cual fue recientemente resuelto por Zúñiga-Reinoso \& Jerez (en prensa), siguiendo las instrucciones propuestas por Clark \& Crosnier (2000) para las especies de crustáceos descrita en los capítulos de zoología del "Voyage au pole sud", obra donde fue descrita la especie por Blanchard.

En relación a la distribución geográfica, del trabajo de Kulzer (1963) se puede deducir que $N$. multicristata estaría presente en el extremo sur-occidental de la estepa patagónica, entre las localidades de Torres del Paine y Puerto Natales (Magallanes - Chile), en contraste con $N$. fallax que se encontraría en la parte continental del extremo sur-oriental de la estepa, en localidades cercanas a Río Gallegos (Santa Cruz - Argentina). Sin embargo, todas las localidades que Kulzer (1963) entrega para $N$. multicristata, se encuentran muy alejadas de la localidad tipo (Puerto del Hambre, según Solier (1851) y Blanchard (1853). Sin embargo, recientemente se corrigió la localidad tipo, la que correspondería a Bahía Pecket, sector ubicado cerca de la boca oriental del Estrecho de Magallanes (Zúñiga-Reinoso \& Jerez, en prensa). Dado que al parecer hubo un error en la designación morfológica de $N$. multicristata por parte de Kulzer (1963), el objetivo de este trabajo es analizar las descripciones originales de N. multicristata hechas por Solier (1851) y Blanchard (1853), compararlas con el material tipo y determinar si $N$. fallax Kulzer, 1963, corresponde a una especie biológica diferente a $N$. multicristata y si la especie que Kulzer (1963) identificó como N. multicristata corresponde a una especie diferente de $N$. multicristata a la descrita por Solier (1851) y Blanchard (1853).

\section{MATERIALES Y MÉTODOS}

Para dilucidar la identidad de ambas especies, se solicitaron fotografías digitales de alta resolución en vista dorsal, ventral y lateral de los sintipos de $N$. multicristata conservados en el Muséum National d'Histoire Naturelle de Paris y paratipos de N. fallax en el Naturhistorisches Museum Basel.

Los ejemplares de las fotografías se compararon con el dibujo de la placa original de Hombron \& Jacquinot, 1846 y descripciones originales de Solier (1851) y Blanchard (1853) para $N$. multicristata y con las descripciones de Kulzer (1963) para N. fallax y N. multicristata. Tanto las fotografías como las descripciones, se compararon posteriormente con el material depositado en las siguientes colecciones entomológicas: Colecciones de Zoología del Instituto de la Patagonia, Universidad de Magallanes, Punta Arenas, Chile (CZIP); Museo Nacional de Historia Natural, Santiago, Chile (MNNC); Museo de Zoología, Universidad de Concepción, Concepción, Chile (UCCC); Muestra de Insectos del Parque Nacional Torres del Paine, Torres del Paine, Chile (MITP), Laboratorio de Genética y Evolución, Universidad de Chile (GVOL) y Instituto Argentino de Investigaciones de las Zonas Áridas (IADIZA).

Por otra parte, para reforzar si los dos morfos corresponden a especies diferentes, se extrajo la genitalia de los machos.

\section{RESULTADOS}

Nyctelia multicristata Blanchard en Hombron \& Jaquinot, 1846: Pl. 10, Fig. 1. (Fig. 1b y Fig. 3a, b, c y d). Nyctelia multicristata en Solier 1851:141, Blanchard en Dumond d'Urville, 1853: 143, Lacordaire 1859: 169; Gemminger and Harold 1870: 1882 (cat.), Fairmaire 1876: 350 (rev), Howard 1889: 199 (cat), Gebien 1910: 144 (cat.), Bruch 1915: 268 (cat.), Gebien 1937: 749 (cat.), Blackwelder 1945: 518 (cat.).

Nyctelia fallax en Kulzer, 1963: 57. Nueva sinonimia. Peña 1966: 416 (cat.); Flores 1997: 10 (list).

\section{DESCRIPCIÓN}

CABEZA: Frente glabra, con puntuación abundante y gruesa. Ojos con setas cortas ventralmente. Clipeo con bordes laterales provistos de abundantes setas cortas las cuales se hacen más esparcidas en la base de la parte proximal. Mentón con setas cortas, levemente esparcidas. Gula con rugosidad suave.

Tórax: Pronoto dos veces más ancho que largo siendo más ancho en la base. Disco glabro y débilmente punteado en los márgenes laterales. Margen notorio, fuertemente almenado, con 4 a 6 estrías diagonales situadas en la base. Proepisterno con pilosidad variable (abundante, escasa o glabros). Proceso prosternal rugoso. Meso y metasterno rugoso.

ÉLitros: Semicóncavos, glabros y fuertemente estriados. Sutura elitral acanalada muy notable en los ejemplares de gran tamaño y menos notoria en individuos de menor tamaño (i.e escudo canaliculado). Estriación muy variada, tanto en número, como en diseño, presentando todas las formas y direccionalidad de estrías que se describiran posteriormente. Margen elitral fuertemente crenulado, se extiende hacia el ápice de los élitros. Apófisis con setas cortas, rectas y dispersas, a veces muy escasas.

PATAS: De color variable (negras, rojo oscuro o naranja). Fémures con rugosidad variable. Tibia protorácica dorsalmente punteada, margen exterior y ventral granuloso, cada gránulo con espinas diminutas en el ápice. Tibias meso y metatorácicas con granulación densa en forma triangular, cada gránulo con una espina diminuta. 


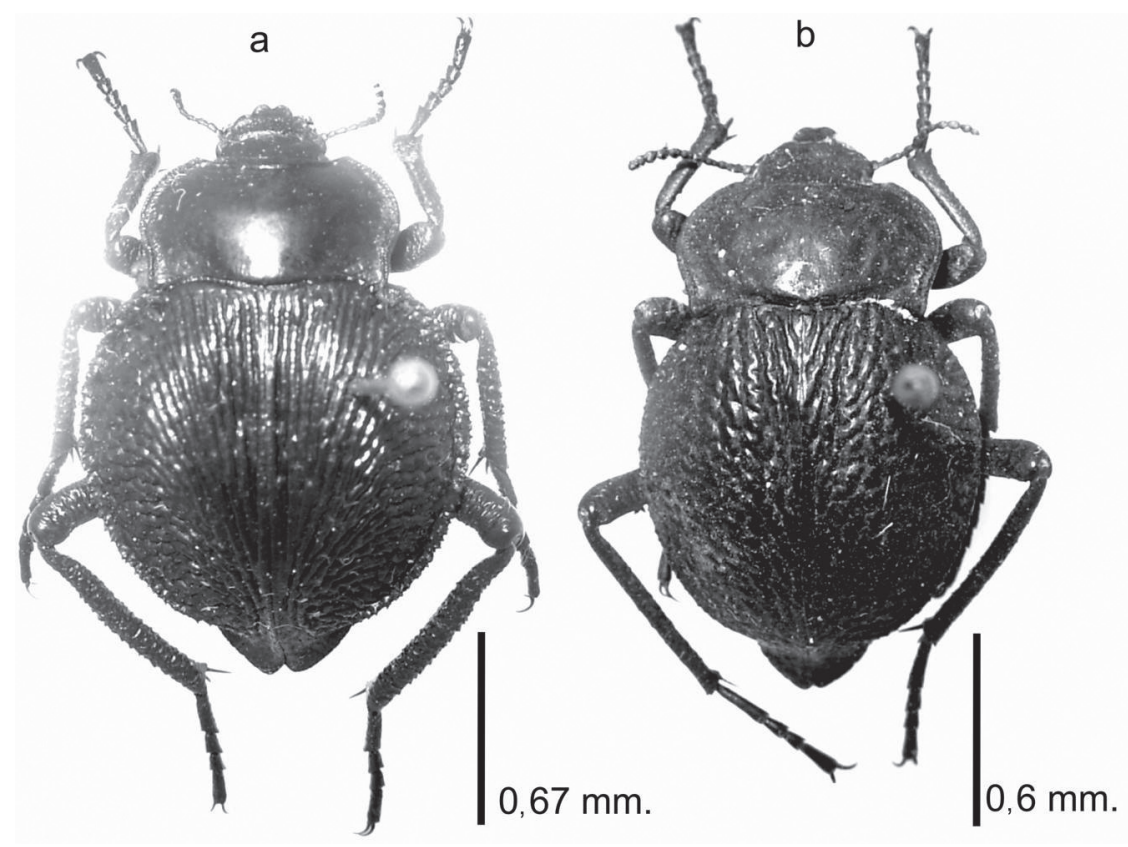

Figura 1. Vista dorsal de ejemplares machos de Nyctelia multicristata Blanchard 1846 (a) y Nyctelia confusa Zúñiga-Reinoso n. sp. (b).

FiguRE 1. Dorsal view from male specimens of Nyctelia multicristata Blanchard 1846 (a) and Nyctelia confusa Zúñiga-Reinoso sp. n. (b).

Abdomen: Glabro, con el ventrito I y II (a veces el III en ejemplares grandes) con estriaciones leves. Ventrito III (cuando corresponda), IV y V lisos.

Genitalia masculina: Edeago con los parámeros del tegmen rectos, aguzados en el extremo distal; lámina basal del tegmen con forma elíptica, margen basal ventralmente triangular, con setas apicales en la superficie ventral. Lóbulo medio recto, con el foramen apical amplio, base cuadrada y ápice redondeado. Láminas basales del tegmen con base convexa. (Fig. 2 a).

\section{Material Examinado}

Material tipo DE $N$. multicristata: 1 Sintipo (fotografía). Etiquetas: Magellan, Port-Famine (Puerto del Hambre).col. Jaquinot 1841. N. multicristata. det. B. J. MNHN EC1731. 2 Sintipos (fotografías). Magellan. col. de Montravel 1841. N. multicristata. det. Blanchard. MNHN EC1732 y MNHN EC1733.

Material tipo de $N$. fallax: 1 Paratipo (fotografía). Argentina. Santa Cruz. Río Chico (Río Gallegos). 26.X.1960. col. L. Peña. Nyctelia fallax n. sp. det Kulzer 1962. 2 Paratipos (fotografía). Argentina. Santa Cruz. Río Chico. X.1960. col. Peña. Nyctelia fallax n. sp. det Kulzer 1962.

OtRO MATERIAL EXAMINADO:

Material Seco: Chile, Magallanes, Gallegos chico.
18.X.1970. col. B. Markham. 1 ejemplar (CZIP). Chile, Magallanes, Laguna Timone. 27.XII.1973. col. D. Lanfranco. 16 ejemplares (CZIP). Chile, Magallanes, Parque Nacional Pali Aike. 4.XII.1974. col. C. Mancilla. 1 ejemplar (CZIP). Chile, Magallanes, Parque Nacional Pali Aike. 25/26.XI.1975. col. J. Petersen. Nyctelia fallax Kulzer 1963. det. G. Flores. 1 ejemplar (CZIP). Chile, Magallanes, Parque Nacional Pali Aike. 25/26.IX.1975. col. J. Petersen. 1 ejemplar (CZIP). Chile, Magallanes, Parque Nacional Pali Aike. 14.XI.1980. col. D. Lanfranco. Nyctelia fallax Kulzer 1963. det. G. Flores. 1 ejemplar (CZIP). Chile, Magallanes, Parque Nacional Pali Aike. 15.X.2002. col. A. Zúñiga. 8 ejemplares (UCCC). Chile, Magallanes, Parque Nacional Pali Aike. 15.X.2003. col. A. Zúñiga. 7 ejemplares (UCCC). Chile, Magallanes, Parque Nacional Pali Aike. Sector brazo norte 2.XI.1975. col. M. Martinic. 3 ejemplares (CZIP). Chile, Magallanes, estancia Brazo Norte. 24.X.1980. col. D. Lanfranco. Nyctelia fallax Kulzer 1963. det. G. Flores. 1 ejemplar (CZIP). Chile, Magallanes, estancia Brazo norte. 24.X.1980. col. J. Petersen. 3 ejemplares (CZIP). Chile, Magallanes, Laguna Blanca. 18.XI.1972. col. E. Couve. Nyctelias bremei (Wat). det. L. Peña. 1 ejemplar (CZIP).Chile, Magallanes, Ruta Y-405. 10.XII.2003. col. A. Zúñiga. 4 ejemplares (UCCC). Chile, Magallanes, Punta del Monte. 10.XII.2002. col. A. Zúñiga. 1 ejemplar (UCCC). Chile, Magallanes, Monte Alto. 14.XII.1960. col. T. Cekalovic. 2 ejemplares (UCCC). 
Material húmedo: Chile, Magallanes. Monte Aymond. 12XII-2009. col. A. Zúñiga. 7 ejemplares (GVOL). Chile, Magallanes. Cruce Oazy Harbour. 12-XII-2009. col. A. Zúñiga. 11 ejemplares (GVOL). Chile, Magallanes, cruce ruta 9/ ruta y-405 (cruce a Gallegos Chico). 7-I-2010. col. A. Zúñiga-G. Gallegos. 10 ejemplares (GVOL). Chile, Magallanes, Punta del Monte. 8-I-2010. col. A. Zúñiga/G. Gallegos. 20 ejemplares (GVOL). Chile, Magallanes, cruce ruta 9/ ruta y-405 (cruce a Gallegos Chico). 2-I-2010. col. A. Zúñiga-G. Gallegos. 10 ejemplares (GVOL).

\section{Nyctelia confusa Zúñiga-Reinoso n. sp.}

(Fig. 1b y Fig. 3e, f, g y h).

Nyctelia multicristata en Kulzer 1963:lamina V: figura 7; Peña 1966: 416 (cat.); Flores 1997: 10 (list); Arias 2000: 129. Mala identificación.

Nyctelia granulata en Arias 2000: 129. Mala identificación.

DESCRIPCIÓN

Cabeza: Clipeo con setas esparcidas cortas de color blanco, situadas laterobasalmente, más intensas en la base del clípeo, reduciéndose en número hacia la parte anterior, bordes laterales con abundantes setas, parte central glabra. Frente glabra y con abundantes puntuaciones gruesas. Mentón con setas cortas, levemente esparcidas. Gula con rugosidad suave y setas cortas bajo el ojo.

Tórax: Pronoto más ancho que largo, siendo más ancho en la base. Sin puntuaciones en el disco. Fuertemente punteado en el borde lateral anterior, particularmente en las prolongaciones anteriores del pronoto, las que se reducen paulatinamente en número y tamaño hacia el disco. Borde lateral posterior con 4 a 6 estrías diagonales hacia la base del pronoto. Margen del pronoto conspicuo, levemente almenado en los bordes laterales. Proepisterno con pilosidad variable (abundante, escasa o glabros). Proceso prosternal Punteado o rugoso. Meso y metasterno rugosos.

Élitros: Cóncavos, glabros y fuertemente estriados. Estriación muy variada tanto en número, como en diseño, presentando todas las formas y direccionalidad de estrías (ver abajo). Borde lateral fino y en algunas ocasiones poco notorio, pudiendo ser almenado y/o levemente crenulado. Presente en la mitad anterior de los élitros. Apófisis con setas cortas, rectas y dispersas por toda la estructura.

Abdomen: Glabro, con el ventrito I con estriaciones leves. Ventrito II, con estriaciones a penas notorias. Ventritos III, IV y $\mathrm{V}$ lisos.

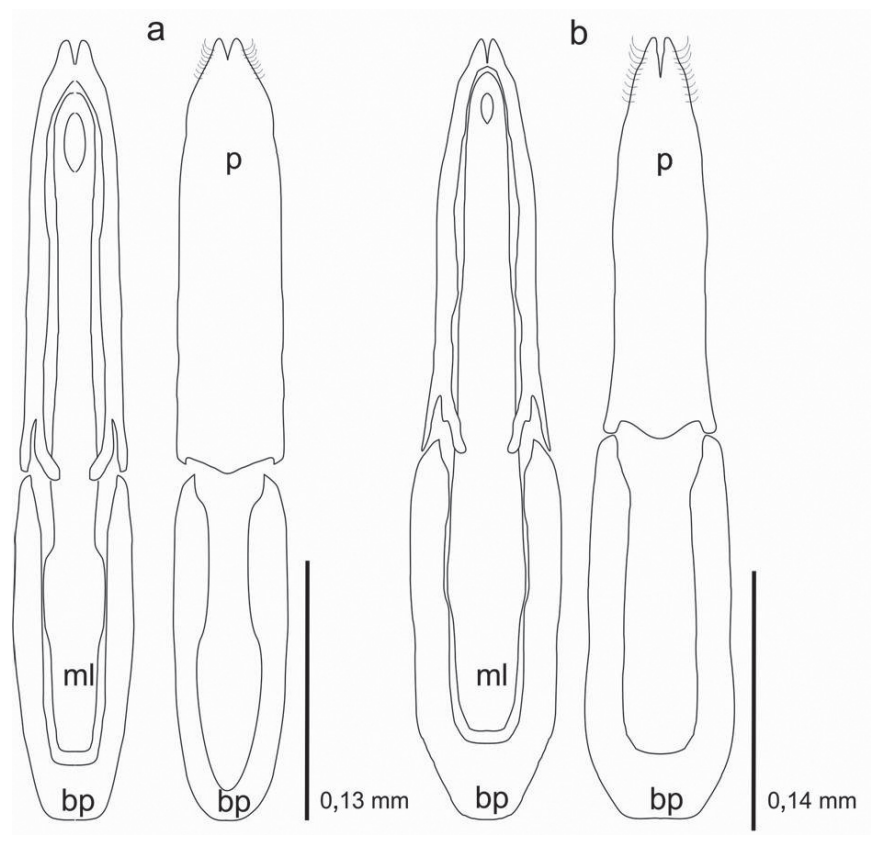

Figura 2. Genitalia macho de las especies de Nyctelia en vista dorsal y ventral. Nyctelia multicristata Blanchard 1846 (a y b) y Nyctelia confusa Zúñiga-Reinoso n. sp. (c y d). bp: lamina basal del tegmen, p: parameros del tegmen, ml: lobulo medio.

Figure 2. Male Genitalia Nyctelia species in dorsal and ventral view. Nyctelia multicristata Blanchard 1846 (a) and Nyctelia confusa Zúñiga-Reinoso sp. n. (b). bp: basal lamina of tegmen, p: parameres of tegmen, ml: middle lobe. 
PatAS: De color variable (negras, rojo oscuro o naranja). Fémures lisos o presentan leves ondas. Tibias meso y metatorácicas con gránulos pequeños y dispersos. Cada gránulo con puntas diminutas. Tibia protorácica dorsalmente punteada, borde exterior granuloso, ventralmente granuloso y con espinas diminutas.

Genitalia masculina: Edeago con los parámeros del tegmen aguzándose desde la base hacia el ápice. Margen basal ventralmente bisinuado, con setas apicales en la superficie ventral. Lóbulo medio más ancho en la base, aguzándose hacia el ápice, con la apertura apical estrecha, base cuadrada, punta redonda. La mitad del ancho de los parámeros del tegmen. Láminas basales del tegmen con base rectangular. (Fig 2 b)

Etimología: Llamada confusa, debido a la confusión que tuvo Kulzer (1963) al asignar a esta forma como $N$. multicristata y a la compleja historia taxonómica de la especie.

Material tiPo: Holotipo, macho: Chile, Última Esperanza, Parque Nacional Torres del Paine, Laguna Amarga. 15-I200. col. A. Zúñiga. det. A. Zúñiga. MZUC - UCCC 35723. Alotipo, hembra: Chile, Última Esperanza, Parque Nacional Torres del Paine, Laguna Amarga. 16-I-2004. col. A. Zúñiga. MZUC - UCCC 35724. Paratipos 372 ejemplares:

Paratipo: Chile, Última Esperanza, la Porfiada. 20/30. XII.1978. col. W. Sielfield. 4 ejemplares (CZIP).Chile, Última Esperanza, cerro León. 10.I.1955. col. T. Cekalovic. 1 ejemplar (UCCC). Chile, Última Esperanza, estancia Cerro Guido. 7.I.1955. col. T. Cekalovic. 11 ejemplares (UCCC). Río Las Chinas, Ultima Esperanza. Leg. Cekalovic, 2-XI1952. 4 ejemplares (IADIZA).Chile, Última Esperanza, Parque Nacional Torres del Paine, Laguna Azul. sin fecha. col. F. Barrientos. 3 ejemplares (MITP). Parque Nacional Torres del Paine, Laguna Azul. 2.II.1952. col. T. Cekalovic. 1 ejemplar (UCCC). Chile, Última Esperanza, Parque Nacional Torres del Paine, Laguna Azul. 16.X.1981. col. L. Peña. 1 ejemplar (UCCC). Chile, Última Esperanza, Parque Nacional Torres del Paine, Laguna Azul. 23.I.1981. col. D. Lanfranco. 1 ejemplar (CZIP). Chile, Última Esperanza, Parque Nacional Torres del Paine, Laguna Azul. 23.I.1981. col. J. Petersen. 1 ejemplar (CZIP). Chile, Última Esperanza, Parque Nacional Torres del Paine, Laguna Azul. 9.XII.2004. col. A. Zúñiga. 15 ejemplares (UCCC). Chile, Parque Nac. Torres del Paine, Laguna Azul. 9.I.2004. col. A. Zúñiga. 2 ejemplares (IADIZA). Laguna Azul, Magallanes, 16.X.1981. col. L. Peña. 1 ejemplar (IADIZA). Laguna Amarga, Natales, Magallanes. 14-20.XII.1960, col. L. Peña. 5 ejemplares (IADIZA). Chile, Última Esperanza, Parque Nacional Torres del Paine, Laguna Amarga. 8.XI.1962. col. T. Cekaovic. 7 ejemplares (UCCC). Chile, Última Esperanza, Parque Nacional Torres del Paine, Laguna Amarga. 10.II.1979. col. D. Lanfranco J. Petersen. 2 ejemplares (CZIP). Chile, Última Esperanza, Parque Nacional Torres del Paine, Laguna Amarga. 10.II.1981. col. D. Lanfranco. 1 ejemplar (CZIP). Chile, Última Esperanza, Parque Nacional Torres del Paine, Laguna Amarga. 5.I.2003. col. J. Cárdenas. 1 ejemplar (UCCC). Chile, Última Esperanza, Parque Nacional Torres del Paine, Laguna Amarga. 4.I.2004. col. A. Zúñiga. 1 ejemplar (UCCC). Chile, Última Esperanza, Parque Nacional Torres del Paine, Laguna Amarga. 6.I.2004. col. A. Zúñiga. 1 ejemplar (UCCC). Chile, Última Esperanza, Parque Nacional Torres del Paine, Pudeto. 19.II.1974. col. E. Pisano. 1 ejemplar (CZIP). Chile, Última Esperanza, Parque Nacional Torres del Paine, laguna de los Cisnes. 7.II.1979. col. D. Lanfranco- J. Petersen. 2 ejemplares (CZIP). Chile, Última Esperanza, Parque Nacional Torres del Paine, lago Sarmiento. 26.I.1981. col. E. Pisano. 9 ejemplares (CZIP). Chile, XII Región, U. Esperanza, Parque Nac. Torres del Paine, Lago Sarmiento. 28.I.1998. col. G. Flores. 1 ejemplar (IADIZA). Chile, Última Esperanza, Parque Nacional Torres del Paine, vega Puma. I.2006. col. A. Zúñiga. 3 ejemplares (UCCC). Chile, Última Esperanza, Parque Nacional Torres del Paine. 7.I.2004. col. A. Zúñiga. 17 ejemplares (UCCC). Chile, Última Esperanza, Parque Nacional Torres del Paine. 8.I.2004. col. A. Zúñiga. 6 ejemplares (UCCC). Chile, Última Esperanza, Parque Nacional Torres del Paine. 14.I.2004. col. A. Zúñiga. 5 ejemplares (UCCC). Chile, XII Región, Parque Nac. Torres del Paine. 10.I.1995. col. G. Flores. 1 ejemplar (IADIZA). Chile, XII Región, Parque Nac. Torres del Paine. 10.I.1995. col. G. Debandi. 1 ejemplar (IADIZA). Chile, Última Esperanza, Parque Nacional Torres del Paine. 15.I.2004. col. A. Zúñiga. 15 ejemplares (UCCC). Chile, Última Esperanza, río de las Chinas. 2.IX.1952. col. Rodríguez. 9 ejemplares (UCCC). Chile, Última Esperanza, Rincón Negro. 22.I.1966. col. T. Cekalovic. 7 ejemplares (UCCC). Chile, Última Esperanza, Puerto Natales. 7.II?.1952. col. Rodríguez. 61 ejemplares (UCCC).

PARAtipo: Chile, Última Esperanza, Parque Nacional Torres del Paine, camino laguna Amarga. 20.XII.2010. col. A. Zúñiga. 6 ejemplares (GVOL). Chile, Última Esperanza, Parque Nacional Torres del Paine, laguna Sarmiento. 20.XII.2010. col. A. Zúñiga. 15 ejemplares (GVOL). Chile, Última Esperanza, Parque Nacional Torres del Paine, laguna de los Cisnes. 20.XII.2010. col. A. Zúñiga. 7 ejemplares (GVOL). Chile, Última Esperanza, Parque Nacional Torres del Paine, Portería Sarmiento. 20.XII.2010. col. A. Zúñiga. 13 ejemplares (GVOL). Chile, Última Esperanza, Torres del Paine, cruce Amarga-Sarmiento. 20.XII.2010. col. A. Zúñiga. 12 ejemplares (GVOL). Chile, Última Esperanza, Parque Nacional Torres del Paine, camino laguna Azul. 21.XII.2010. col. A. Zúñiga. 20 ejemplares (GVOL). Chile, Última Esperanza, cruce Torres del Paine- Cerro Guido. 
21.XII.2010. col. A. Zúñiga. 12 ejemplares (GVOL). Chile, Última Esperanza, puente río de la Chinas. 21.XII.2010. col. A. Zúñiga. 5 ejemplares (GVOL). Chile, Última Esperanza, Sierra Leona. 21.XII.2010. col. A. Zúñiga. 10 ejemplares (GVOL). Chile, Última Esperanza, camino estancia el Lazo. 22.XII.2010. col. A. Zúñiga. 12 ejemplares (GVOL). Chile, Última Esperanza, camino bahía el Bote. 22.XII.2010. col. A. Zúñiga. 10 ejemplares (GVOL). Chile, Última Esperanza, laguna Figueroa. 22.XII.2010. col. A. Zúñiga. 10 ejemplares (GVOL). Chile, Última Esperanza, Cruce laguna Sofía. 23.XII.2010. col. A. Zúñiga. 13 ejemplares (GVOL). Chile, Última Esperanza, Monumento Natural Cueva del Milodón. 24.XII.2010. col. A. Zúñiga. 3 ejemplares (GVOL). Argentina, Santa Cruz, Río Turbio. 18.XII.2010. col. A. Zúñiga. 21 ejemplares (GVOL). Argentina, Santa Cruz, $15 \mathrm{~km}$ al sur de Cancha Carrera. 18.XII.2010. col. A. Zúñiga. 7 ejemplares (GVOL).

\section{DISCUSION}

A partir de las observaciones, se puede establecer que las características del pronoto (puntuación escasa o abundante y esparcida), la forma del escudo (canaliculado o no canaliculado), el borde elitral (carinado o almenado) y las patas (granulosas o levemente granulosas), además de la forma de la genitalia de los machos, son caracteres constantes que permiten diferenciar dos morfos. Al comparar las descripciones originales de $N$. multicristata de Solier (1851) y Blanchard (1853) y con los fototipos de $N$. multicristata y $N$. fallax, se puede establecer que los caracteres registrados coinciden en su totalidad con ambos tipos. Sin embargo, al comparar estas descripciones con los morfos que Kulzer (1963) designa como N. multicristata y $N$. fallax, podemos establecer que coinciden en su totalidad sólo con el morfo de $N$. fallax. Por lo tanto, el morfo que según Kulzer (1963) corresponde a $N$. multicristata, posee a lo menos tres caracteres que lo separan de las descripciones originales de Solier (1851) y Blanchard (1853). Por otra parte, el análisis de la genitalia de los machos, muestra que el morfo designado por Kulzer (1963) como " $N$. multicristata" presentan edeago con estilos laterales aguzados hacia el ápice y la lámina basal del tegmen con forma rectangular (Fig. 2b). Estos ejemplares presentan un edeago diferente a los insectos designados por Kulzer (1963) como “ $N$. fallax", los que presentan edeago con estilos laterales rectos, aguzados en el extremo distal y las láminas basales del tegmen con forma elíptica (Fig. 2 a). Finalmente, ambos morfos presentan una gran variabilidad en el diseño elitral, encontrándose a lo menos tres formas de estrías: 1) completamente lisas, 2) completamente rugosas y 3 ) rugosas en la parte proximal y más lisas hacia el ápice. En relación a la dirección de las estrías, se pueden clasificar como: 1) Estrías longitudinales rectas desde la zona proximal del élitro hacia el ápice, 2) estrías curvas, desde las epipleuras hacia el ápice, 3) una combinación de ambas y 4) estrías poco definidas, que le dan al disco un aspecto granuloso. Toda esta variabilidad puede darse tanto dentro de una misma localidad, como entre localidades diferentes. Por lo que el diseño elitral no deben ser utilizados para separar ambas especies.

Según las observaciones, la especie que Kulzer (1963) describió como $N$. fallax basándose solo en la forma y dirección de la estriación elitral, es una variación morfológica de $N$. multicristata, por lo cual se la debe considerar como sinónimo junior. Al respecto, y dentro de una misma localidad, la dirección y forma de las estrías, así como el color de las patas son altamente variables, por lo cual no constituyen caracteres diagnósticos.

Por otra parte, la especie que Kulzer (1963) nominó como $N$. multicristata, al menos morfológicamente (i.e morfología externa y genitalia) corresponde a una nueva especie, denominada aquí como $N$. confusa. En su descripción incluimos algunos caracteres descritos por Kulzer (1963) para N. multicristata y la descripción de las estructuras de la genitalia masculina propuesta por Flores (1996), como un carácter diagnóstico para las especies del género. La serie examinada de ejemplares de la especie $N$. confusa, proveniente de la parte norte de la distribución de la especie, entre las localidades de Torres del Paine y Sierra Baguales, permitió establecer la constancia de los caracteres diagnósticos entregados en este trabajo. Sin embargo, en la zona de Cerro Castillo, Puerto Natales y Río Turbio, se encuentran individuos con el cuerpo levemente más aplanado y élitros con estrías más profundas, las que fueron asignadas como una variación morfológica al sur de la distribución de la especie. Análisis poblacionales más exhaustivos, podrán entregar más antecedentes acerca de esta variabilidad morfológica.

Finalmente este trabajo aporta al esclarecimiento de una confusión histórica en la designación de las especies tratadas, lo que ha conllevado a un desorden generalizado de catálogos y colecciones. Esto se debe principalmente a la antigüedad de los trabajos originales y a la falta de expertos que se interioricen en la ardua tarea taxonómica y en los costos de largo plazo de los aspectos históricos de este grupo.

\section{AGRADECIMIENTOS}

El autor agradece especialmente a Antoine Mantilleri y a Isabelle Zürcher-Pfander por el envio de las fotografías de los tipos depositados en el Muséum National d'Histoire Naturelle de Paris y Naturhistorisches Museum Basel respectivamente. 


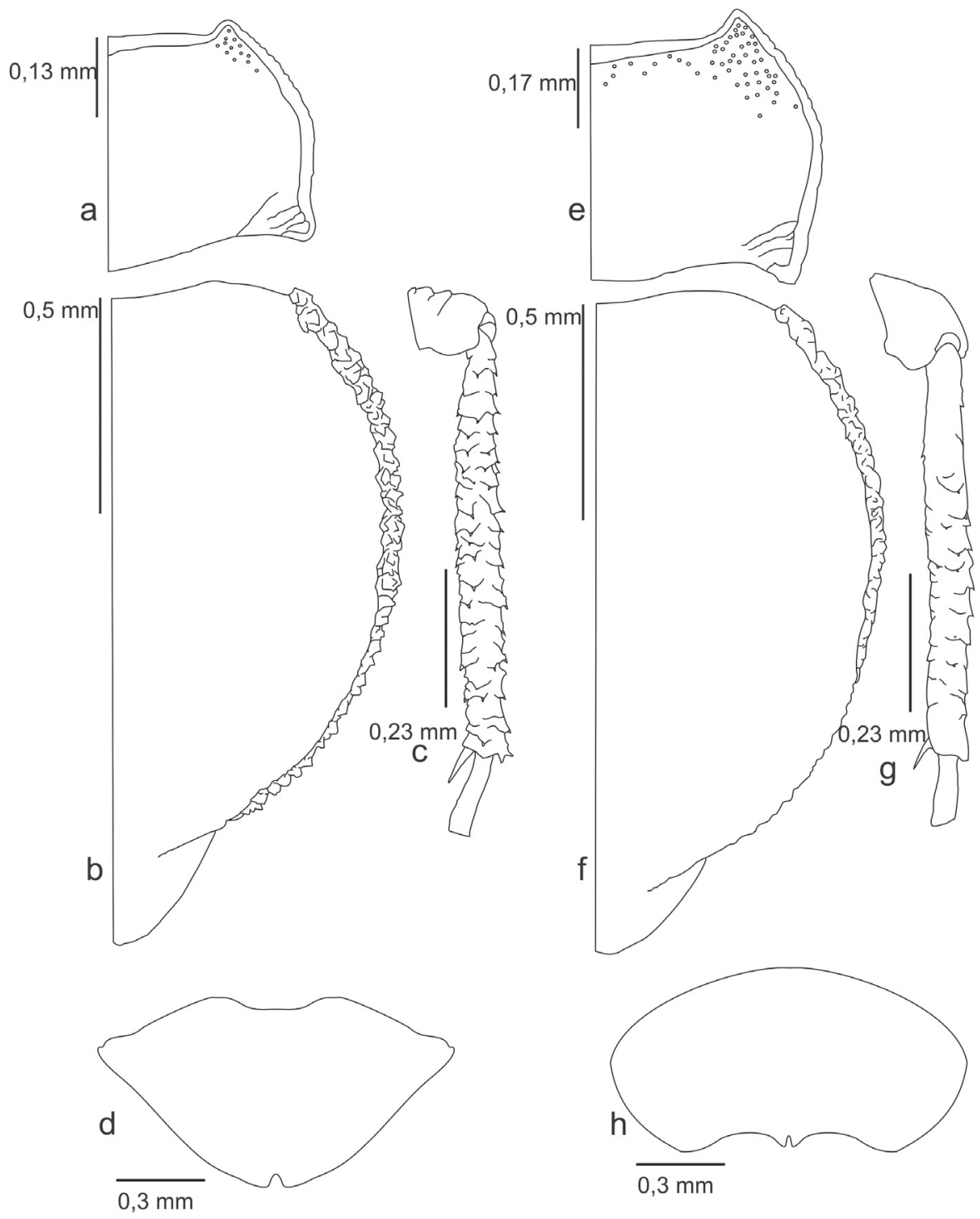

Figura 3. Detalles morfológicos diagnósticos para las especies Nyctelia multicristata Blanchard 1846 (a, b, c y d) y Nyctelia confusa Zúñiga-Reinoso n. sp. (e, f, g y h). a-e: detalles del pronoto, b-f: detalles del margen elitral, c-g: vista dorsal de femur y tibia metatorácica, d-h: vista posterior de los élitros.

Figure 3. Details morphological diagnostic for species Nyctelia multicristata Blanchard 1846 (a, b, c and d) and Nyctelia confusa ZúñigaReinoso sp. n. (e, f, g and h). a-e: details of the pronotum, b-f: elytral margin details, c-g: dorsal view of femur and tibia metatorácica, d-h: back view of elytra. 
Por otra parte, también agradecer a Mario Elgueta del Museo Nacional de Historia Natural, a Jorge Artigas Museo de Zoología de la Universidad de Concepción, a Sergio Roig Juñent del Instituto Argentino de Zonas Aridas y a Vicente Pérez del Instituto de la Patagonia, quienes pusieron a disposición las colecciones para ser revisadas. Además agradecer a Gustavo Flores por las fotografías enviadas de los ejemplares depositados en IADIZA, a Eduardo Faúndez y. Gloria Gallegos, por los datos entregados de la CZIP. También agradecer a Alejandra Silva e Irene Ramírez de CONAF Magallanes y Puerto Natales respectivamente, por gestionar los permisos para el ingreso a Áreas Silvestres protegidas de la Región. Finalmente agradecer a la beca CONICYT No 21110367 para estudios de Doctorados Nacionales. y al proyecto DIUC No 208.112.99 -3.

\section{BIBLIOGRAFIA}

Arias, E. 2000. Coleópteros de Chile. Fototeknika. Santiago, Chile. 209 pp.

Blackwelder, R. E. 1945. Checklist of the coleopterous insects of Mexico, Central America, the West Indies and South America. Part 3. Bull. U.S. Natl. Mus.185: 343-550.

Blanchard, E. 1853. Zoologie: Description des Insectes p. 143. En: Dumont d'Urville J.S. Voyage au pole sud et dans l'Océanie sur les corvettes l'Astrolabe et la zélée: exécuté par ordre du Roi pendant les annes 1837-1838-1839-1840. Tomo IV. 422 pp.

Clark P.F. \& A. Crosnier. 2000. The Zoology of the Voyage au pole sud et dans l'Océanie sur les corvettes l'Astrolabe et la Zélée exécuté par ordre du roi pendant les années 1837-1838-1839-1840 sous le commandement de M. Dumont-D'Urville, 1842-1854: titles, volumes, plates, text, contents and proposed dates and anecdotal history of the publication. Archives of Natural History. 27: 407-437.

Fairmaire, L. 1876. Révision des coléoptéres du Chili. Famille des Tenebrionidae, Tribu des Nyctélites. Annales de la Société Entomologique de France 5: 143-170,341-383.

FLoRES, G.E. 1996. Estudio comparativo de las estructuras genitales en la tribu Nycteliini (Coleoptera: Tenebrionidae). Revista de la Sociedad Entomológica Argentina 55: 33-48.

Flores, G.E. 1997. Revisión de la tribu Nyctelini (Coleoptera: Tenebrionidae). Revista de la Sociedad Entomológica Argentina 56: 1-19.

FLores, G. E. 2007. New synonymies and lectotype designations in the Neotropical tribes Nycteliini, Praocini, and Scotobiini (Coleoptera: Tenebrionidae).Annales Zoologici 57: 409426.
Flores G.E. 2009. Revision of some types of the South American tribes Nycteliini, Praocini, and Scotobiini (Coleoptera: Tenebrionidae), with new synonymies. Zootaxa 1985: 2133.

Flores, G.E. \& R. CARrara. 2006. Two new species of Nyctelia Latreille (Coleoptera: Tenebrionidae) from western Argentinean high mountains. Annales Zoologici 56: 487495.

Gebien, H. 1910. Tenebrionidae. I: 1-166, II: 167-354. Coleoptm Cat. 18, plates 15,22. Berlín.

Gebien, H. 1937. Katalog der Tenebrioniden. Teil I. Publ. Mus. Entomol. Pietro Rossi Duino 2: 505-883

Gemminger, M., \& E. Von Harold. 1870. Catalogus Coleopterorum hucusque descriptorum synonymicus et systematicus. Vol. 7: 1801-2180. Monachii.

Hombron, J. B. \& H. JacQuinot. 1846. Insecta: Coleoptera. Atlas d'Histoire Naturelle. Zoologie. Voyage au Pôle Sud et dans l'Océanie sur les corvettes l'Astrolabe et la Zélée pendant les années 1837-1838-1839-1840. pls. 10.

HowARD, L. 1889. Scientific Results of Explorations by the U. S. Fish Commission Steamer Albatross. No. V. Annotated Catalogue of the Insects Collected in 1887-88. Proceedings of the United States National Museum.12: 185-207.

Kulzer, H. 1963. Revision der südamerikanischen Gattung Nyctelia Latr. (Col. Teneb.) (24 Beitrag zur Kenntnis der Tenebrioniden). Entomologische Arbeiten aus dem Museum George Frey 14: 1-71.

Latreille, P.A. 1825 Famillies Naturelles du Régne Animal. J. B. Baillière Libraire, Paris. 570 pp.

PeÑA, L. 1963. Las Nyctelias (Coleoptera, Tenebrionidae). Entomologische Arbeiten aus dem Museum George Frey 9:72-75.

Peña, L. E. 1966. Catálogo de los Tenebrionidae (Coleoptera) de Chile. Entomologische Arbeiten aus dem Museum George Frey 17: 397-453.

Roig-Juñent, S. \& G. Flores. 2001. Historia biogeográfica de las áreas áridas de América del sur austral. En: Introducción a la biogeografía en Latinoamérica: Teorías, conceptos, métodos y aplicaciones (Eds. Llorente, J. \& J. Morrone.). 277 pp. Las Prensas de Ciencias, Facultad de Ciencias, UNAM. México.

Solier, J. 1851. Insectos: Coleópteros. En: Gay, C. Historia Física y Política de Chile. Zoología. Tomo 5. 563 pp.

Vidal, P. \& M. Guerrero. 2007. Los Tenebriónidos de Chile. Ediciones Universidad Católica de Chile. Santiago, Chile. $478 \mathrm{pp}$.

ZúÑIgA-Reinoso, A. \& V. Jerez. Problemas con la nomenclatura y la localidad tipo de Nyctelia multicristata (Coleoptera: Tenebrionidae). Anales del Instituto de la Patagonia. (en prensa). 